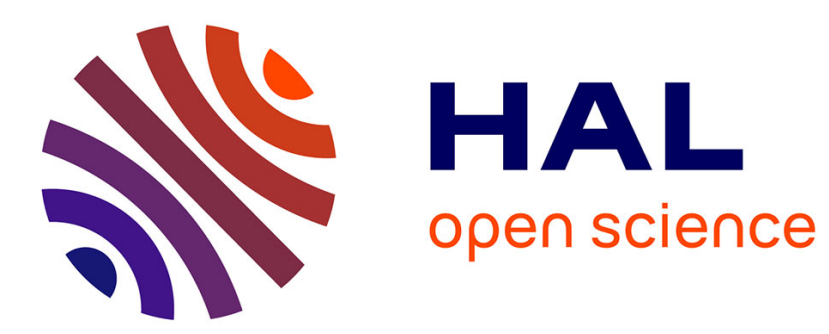

\title{
Distributed Management of Massive Data: an Efficient Fine-Grain Data Access Scheme
}

\author{
Bogdan Nicolae, Gabriel Antoniu, Luc Bougé
}

\section{To cite this version:}

Bogdan Nicolae, Gabriel Antoniu, Luc Bougé. Distributed Management of Massive Data: an Efficient Fine-Grain Data Access Scheme. VECPAR '08: Proceedings of the 8th International Conference on High Performance Computing for Computational Science, Jun 2008, Toulouse, France. pp.532-543, 10.1007/978-3-540-92859-1_47 . inria-00323248

\section{HAL Id: inria-00323248 \\ https://hal.inria.fr/inria-00323248}

Submitted on 12 Oct 2008

HAL is a multi-disciplinary open access archive for the deposit and dissemination of scientific research documents, whether they are published or not. The documents may come from teaching and research institutions in France or abroad, or from public or private research centers.
L'archive ouverte pluridisciplinaire HAL, est destinée au dépôt et à la diffusion de documents scientifiques de niveau recherche, publiés ou non, émanant des établissements d'enseignement et de recherche français ou étrangers, des laboratoires publics ou privés. 


\title{
Distributed Management of Massive Data: an Efficient Fine-Grain Data Access Scheme
}

\author{
Bogdan Nicolae ${ }^{1}$, Gabriel Antoniu ${ }^{2}$, and Luc Bougé ${ }^{3}$ \\ 1 University of Rennes 1/IRISA, Campus de Beaulieu, 35042 Rennes Cedex, France \\ bogdan.nicolae@inria.fr \\ 2 INRIA/IRISA, Campus de Beaulieu, 35042 Rennes Cedex, France \\ gabriel antoniu@inria.fr \\ 3 ENS Cachan Brittany/IRISA, Campus de Beaulieu, 35042 Rennes Cedex, France \\ luc. bouge@bretagne.ens-cachan.fr
}

\begin{abstract}
This paper addresses the problem of efficiently storing and accessing massive data blocks in a large-scale distributed environment, while providing efficient fine-grain access to data subsets. This issue is crucial in the context of applications in the field of databases, data mining and multimedia. We propose a data sharing service based on distributed, RAM-based storage of data, while leveraging a DHT-based, natively parallel metadata management scheme. As opposed to the most commonly used grid storage infrastructures that provide mechanisms for explicit data localization and transfer, we provide a transparent access model, where data are accessed through global identifiers. Our proposal has been validated through a prototype implementation whose preliminary evaluation on the Grid'5000 testbed provides promising results.
\end{abstract}

\section{Introduction}

Managing data at a large scale is paramount nowadays. Governmental and commercial statistics, climate modeling, cosmology, genetics, bio-informatics, etc. are just a few examples of fields routinely generating huge amounts of data. It becomes crucial to efficiently manipulate these data, which must be shared at the global scale. In such a context, one important goal is to provide mechanisms allowing to manage massive data blocks (e.g., of several terabytes), while providing efficient fine-grain access to small parts of the data. Several types of applications exhibit such a need for efficient scaling to huge data sizes: databases ([1, 2, 3]), data mining [4], multimedia [5], etc.

Towards transparent management of data on the grid. The management of massive data blocks naturally requires the use of data fragmentation and of distributed storage. Grid infrastructures, typically built by aggregating distributed resources that may belong to different administration domains, provide an appropriate solution. When considering the existing approaches to grid data management, we can notice that most of them heavily rely on explicit data localization 
and on explicit transfers of large amounts of data across the distributed architecture: GridFTP [6], Reptor [7], Optor [7], LDR [8], Chirp [9], IBP [10], NeST [1], etc. Managing huge amounts of data in such an explicit way at a very large scale makes the design of grid application much more complex. One key issue to be addressed is therefore the transparency with respect to data localization and data movements. Such a transparency is highly suitable, as it liberates the user from the need to handle data localization and transfers.

However, a few grid data management systems acknowledge that providing a transparent data access model is important by integrating this idea at the early stages of their design. Grid file systems, for instance, provide a familiar, file-oriented API allowing to transparently access physically distributed data through globally unique, logical file paths. The applications simply open and access such files as if they were stored on a local file system. A very large distributed storage space is thus made available to those existing applications that usually use file storage, with no need for modifications. This approach has been taken by a few projects like GFarm [12], GridNFS [13], LegionFS [14], etc.

On the other hand, the transparent data access model is equally defended by the concept of grid data-sharing service [15], illustrated by the JuxMem platform [16]. Such a service provides the grid applications with the abstraction of a globally shared memory, in which data can be easily stored and accessed through global identifiers. To meet this goal, the design of JuxMem leverages the strengths of several building blocks: consistency protocols inspired by DSM systems; algorithms for fault-tolerant distributed systems; protocols for scalability and volatility support from peer-to-peer (P2P) systems. Note that such a system is fundamentally different from traditional DSM systems (such as TreadMarks, etc.). First, it targets a larger scale through hierarchical consistency protocols suitable for an efficient exploitation of grids made of a federation of clusters. Second, it addresses from the very beginning the problem of resource volatility due to failures or to the lack of resource availability.

Compared to the grid file system approach, this approach improves access efficiency by totally relying on main memory storage. Besides the fact that a main memory access is more efficient than a disk access, the system can leverage locality-optimization schemes developed for the DSM consistency protocols.

Limitations. However, the JuxMem grid data-sharing service suffers from some limitations with respect to the efficient storage and access of massive data blocks. Actually, data are not fragmented in JuxMem: each individual data is physically stored as a single data block in the main memory of a storage provider, and possibly replicated as such on multiple backup providers. Consequently, the largest data block that the service is able to store is limited by the size of the RAM of a single provider, typically, a few gigabytes. This lack of fragmentation has another drawback regarding load balancing as all accesses to different parts of the same massive block are served by the same RAM provider.

Recently, the efficient allocation and access of massive data blocks in main memory has been addressed by the JumboMem [17 system. This system is designed for clusters, not for grids. It allows users to manipulate large contiguous 
data blocks (of the order of $1 \mathrm{~TB}$ ) using the aggregated RAM of a set of nodes interconnected through a high-speed Infiniband System Area Network. However, JumboMem is targeted for a single user and does not enable data sharing: it does not provide synchronization, nor replication, nor optimized mechanisms for distributed access by multiple users. In contrast, a lot of applications in the field of databases and data-mining target multi-user environments. This requires adding an efficient concurrency control, which is not natively provided by JumboMem.

Our approach. Our contribution is twofold. First, we propose a data sharing service allowing to store massive blocks of data in a distributed, multi-user environment. Second, efficient fine-grain access to the data is provided thanks to distributed, RAM-based storage of data fragments, while leveraging a DHTbased metadata management scheme, which is natively parallel.

This paper is organized as follows. Section 2 gives an overview of our architecture and describes how data access operations are handled. Section 3 provides a few implementation details and reports on a preliminary experimental evaluation. Finally, on-going and future work is discussed in Section 4 .

\section{Enabling efficient fine-grain access}

Our goal is to provide efficient fine-grain access to massive data blocks stored in large-scale distributed environments such as grids. To goal is addressed in the following way. Data is fragmented into small equally-sized chunks (which will be called pages below) and distributed across the local memory of a large number of grid nodes, which act as providers of storage space. This fragmentation allows: 1) to store huge data blocks; and 2) to avoid contention for disjoint accesses to pages. To each data block, we associate some metadata allowing to identify and localize the pages that belong to that block. In order to avoid contention for metadata access, metadata is structured in a fine-grained manner to be described below, and stored in a distributed hash table (DHT). Finally, efficient large-scale concurrency both for reads and writes is achieved using versioning: concurrent writes to the same page can proceed in parallel on multiple versions of that page. Our contribution lies in the adequate combination of these techniques to achieve efficient fine-grained access to massive data.

\subsection{Architecture}

Our service relies on a set of distributed processes communicating through remote procedure calls (RPCs). In a typical setting, each process is running on a different physical node.

Data providers are responsible for storing and retrieving individual pages in their local RAM.

A versioning manager is responsible for serializing write requests and for directing read requests to the latest version available for reading. 
Metadata providers are responsible for storing information about the identity and localization of the individual pages that make up a data block. In our design, metadata providers are organized as a Distributed Hash Table (DHT). Details are given in Section 2.3.

A provider manager receives and solves the clients' requests for data providers. Available providers must previously register with this entity.

To interact with the service, client processes simply use a client library, to which they pass a list of DHT gateways and the network id (IP address, port) of the versioning manager. The rest of the system is transparent to the clients.

\subsection{User interface}

Clients manipulate massive data blocks through a simple API:

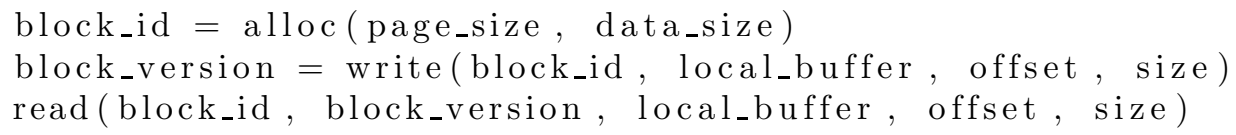

Massive blocks are identified and accessed through a globally unique id, generated when the block is allocated. The user is able to control the granularity (page_size) and maximal size of the block (data_size). Fine-grain access for reads and writes is enabled through (offset, size) range queries. Each write generates a new block version. Read operations may explicitly reference a block version. By default, they return the latest available version.

\subsection{Metadata organization}

Metadata serves the purpose of identifying and localizing the pages corresponding to the range ( offset, size) specified by read and write operations. Our design aims at favoring fast concurrent accesses to metadata.

When the user allocates data_size bytes for a block, the service actually allocates adjusted_size bytes, where adjusted_size is the smallest power of 2 larger than data_size. We organize metadata as a full binary tree. At each level, the nodes of the tree cover disjoint ( offset, size) ranges. The root covers (0, adjusted_size), that is, the whole data block. An intermediate node covering ( offset, size) points to its left child covering (offset, size/2), and to its right child covering ( offset + size $/ 2$, size $/ 2$ ). Leaves cover single pages and point to the page id and to provider holding the page (see Figure 1).

A tree node covering (offset, size) is identified by a key, obtained by applying a hashing function on the tuple (block_id, offset, size, block_version). Intermediate tree nodes store the following information: offset, size, left_key and right_key, which are respectively the keys of its left and right child. Leaves (covering single pages) store a page_id and a provider_id. Tree nodes are stored on the metadata providers using a DHT structure using the keys defined above. This approach is inspired by Merkle trees [18, initially developed to handle Lamport's one-time signatures. 


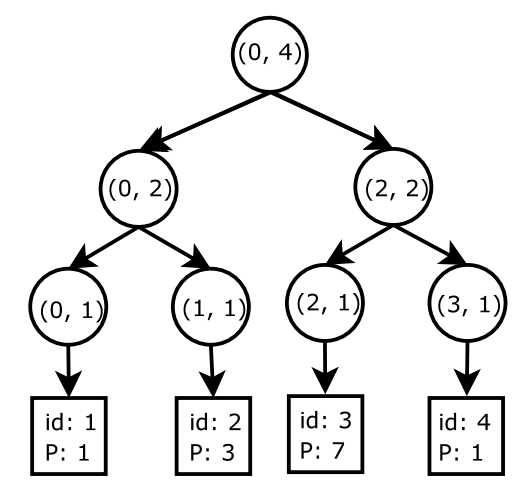

Fig. 1. Metadata representation for a 4-page block. Leaves store page ids Id and the corresponding provider ids P. All nodes are labeled with the (offset, size) range they cover.

By relying on the DHT architecture and by selecting an adequate hashing function, an even distribution of page requests among metadata providers can be guaranteed with a high probability. Each client takes profit of this even distribution by simultaneously contacting a large number of different gateways to the DHT service when executing parallel requests.

\subsection{Managing allocs, reads and writes}

Allocation is the cheapest and simplest operation. The client merely contacts the versioning manager providing a page size and total block size. The versioning manager assigns this block an initial version number, 0 .

To perform a read if no block version is specified, the client (Figure 2(a)) contacts the versioning manager and requests the latest block version available. If a block version is specified by the read operation, then this step is simply skipped. Then, the client contacts the metadata providers and recursively queries the tree nodes covering the range given by ( offset, size) for that particular block version, starting from the root and descending towards the leaves. When a leaf is reached, the client directly contacts the appointed data provider and downloads the actual page. The read operation completes successfully when all the pages have been downloaded. It fails if a node or a page could not be retrieved. In order to enhance parallelism, requests and responses for tree nodes and for pages are handled asynchronously by multiple threads on the client side, and are served in parallel by the various metadata and data providers, respectively.

A write operation (Figure 2(b) initiated by the client completes in several stages.

1. The client contacts the provider manager to retrieve a list of active data providers available to store the pages in (offset, size) to be written. After receiving the reply, it associates a random data provider and a random page 


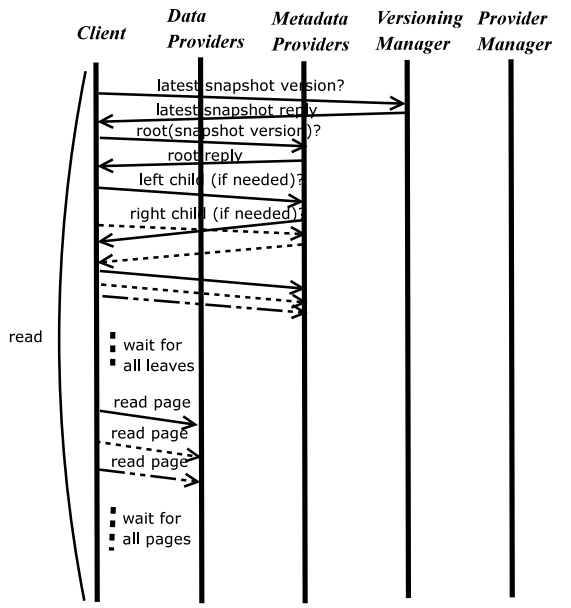

(a) Reading a block: sequence of RPC calls

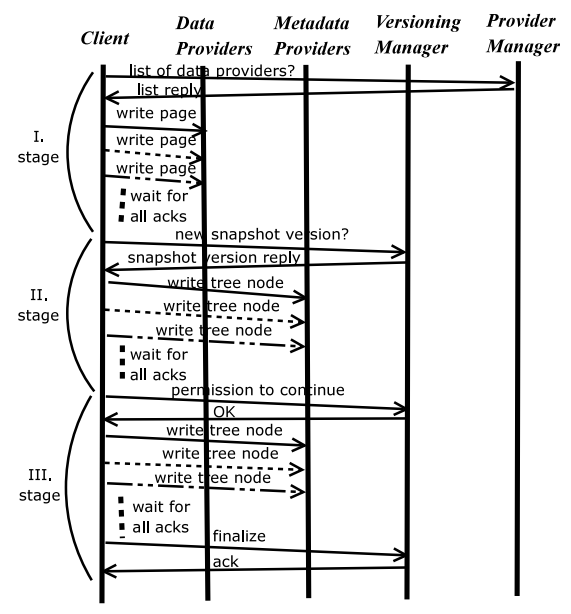

(b) Writes: sequence of RPC calls

Fig. 2. Managing reads and writes: different line styles denote RPCs running in parallel

id to each page, so as to uniquely identify the page in the system with high probability. Then, it contacts the data providers, requesting them to store the pages. As in the case of the read operation, write requests sent to providers are asynchronously handled by the client, and served in parallel by the data providers.

2. After all providers acknowledge that the pages have been stored, the client contacts the versioning manager to receive a new version number which shall identify the new block version. The versioning manager enqueues this write request, marks it as pending and returns the version number to the client. After receiving it, the client generates the corresponding tree nodes with respect to the new block version, starting from the leaves up to this new root. All tree nodes whose range is totally included in the interval [offset, offset + size] are written to the metadata providers. The rest of the nodes are stored for later processing. The goal of this processing is to properly handle concurrent metadata updates for a single block.

3. Then, the client contacts the versioning manager requesting permission to complete the write operation. If this write request is the oldest one in the queue, then the versioning manager grants permission to complete the write. Otherwise it waits for previous pending writes to be dequeued before granting permission. After receiving permission, the client builds the remaining tree nodes. These nodes cover ranges not included in [ offset, offset + size]. They must correctly reference their children corresponding to nodes not modified 
by the current write, by using the latest block version previously completed. At the end of this stage, all generated tree nodes are sent to the metadata providers.

4. Finally, the client confirms write completion to the versioning manager, which dequeues the write and marks its corresponding version as the latest block version.

Note that various versions of the same page may be stored on different providers: for each new page version to be written, the least loaded known provider is chosen for its storage, in order to preserve a global load balance in terms of amount of data stored by the providers. (The precise description of this scheme is out of the scope of this paper.)

An important consequence of this property is that successive incremental versions of a data block can be stored as long as storage space is still globally available in the system: thanks to our choice of preserving a global load balance, a provider will run out of storage space only when all the providers collectively reach their storage limits. In this case, ad-hoc garbage collection can be used to remove the oldest version of the data block. Such a feature has not been implemented in our system, yet.

\section{Implementation and experimental evaluation}

Evaluations are performed using the Grid'5000 19 testbed, a reconfigurable, controllable and monitorable experimental Grid platform spread over 9 sites geographically distributed in France. We use 160 nodes of a Grid'5000 cluster. Each node has a Intel Pentium $4 \mathrm{CPU}$ running at $2.6 \mathrm{GHz}$ under Linux 2.6 (Ubuntu), outfitted with 4 GB of RAM each, and interconnected by a Gigabit Ethernet network. The theoretical maximum network bandwidth is thus $125 \mathrm{MB} / \mathrm{s}$. However, if we consider the IP and TCP header overhead, this maximum becomes slightly lower: $117.5 \mathrm{MB} / \mathrm{s}$ for a MTU of $1500 \mathrm{~B}$. In practice, we could measure $111 \mathrm{MB} / \mathrm{s}$ for a standard TCP socket end-to-end transfer.

\subsection{Implementation details}

We use BambooDHT [20, which provides a stable, scalable DHT implementation on top of which we build the abstraction of our metadata providers and of the provider manager.

The providers and the versioning manager are implemented in $\mathrm{C}++$ using the Boost $\mathrm{C}++$ collection of libraries. We chose Boost for its standardization throughout the $\mathrm{C}++$ community, and for the wide range of functionalities it provides, among which serialization, threading and asynchronous I/O are of particular interest to us.

\subsection{Performance and evaluation}

In this section, we assess the effectiveness of our implementation by running a set of experiments. To support our claim of efficiently dealing with both massive 
blocks and fine-grain access, we fix the allocated block size at $1 \mathrm{~TB}$, and the page size at $64 \mathrm{kB}$ for all our experiments. Thus, the metadata tree generates a significant overhead as the actual data accesses will concern various continuous ranges from $16 \mathrm{MB}$ up to $1 \mathrm{~GB}$ within the overall range of $1 \mathrm{~TB}$.

Using a single client. Our first series of experiments (Figure 3) assesses the overhead of metadata management. We deploy one versioning manager, 100 data providers, and a variable number of metadata providers. Each physical node runs at most one data provider and one metadata provider. A single client writes a series of data, and then reads them back.

It first writes a range size of $16 \mathrm{MB}$ starting from offset 0 . Then, it continues writing a second range of $32 \mathrm{MB}$, starting from the end of the previous range, and so on, doubling the size parameter each time until writing a range of 1 GB. Then, the client successively reads back each of the consecutive segments.

The individual writing and reading times for each segment are logged, sorting out the time used in managing the metadata with respect to the total writing or reading time. Such a cycle is repeated 100 times. This experiment is done for several numbers of metadata providers, that is, several sizes of the DHT, ranging from 5 to 100 .

The average timings are reported on Figure 3. Of course, the larger the DHT, the larger the degree of parallelism in accessing its nodes from the client's threads, whence the shorter the overall time.

These timings show an overhead of $18 \%$ for metadata read operations (Figure 3(a) and $23 \%$ for metadata write operations (Figure 3(b)] for 100 metadata providers. This effectively results in a bandwidth of $92 \mathrm{MB} / \mathrm{s}$ for reads and $86 \mathrm{MB} / \mathrm{s}$ for writes in accessing the final $1 \mathrm{~GB}$ range, to be compared to the maximal limit of $111 \mathrm{MB} / \mathrm{s}$ measured in standard TCP socket end-to-end transfer. On the other hand, using only 5 metadata providers results in a metadata management overhead exceeding $68 \%$, which demonstrates the benefits of using a large number of metadata providers, that is, a large DHT.

Using multiple concurrent clients. Our next series of experiments (Figure \) benchmarks our system in a highly concurrent environment, evaluating its scalability when increasing the number of simultaneous reads and writes. For comparison, we also report on what we call an "ideal" bandwidth corresponding to the aggregation of totally independent read (resp., write) operations. That is, we multiply the bandwidth of a single reader (resp., writer) by the number of readers (resp., writers).

We deploy 80 data providers and 80 metadata providers. Each physical node runs one data provider and one metadata provider. The versioning manager is run on a separate node. Then, we deploy a variable number of clients, each of which being run on a separate node, different from the ones used for data, metadata and versioning manager. Clients are synchronized to start simultaneously. They either read or write a disjoint range of the block: client $i$ uses offset $=i \times 64 \mathrm{MB}$, size $=64 \mathrm{MB}$. For reads, data is prewritten. We measure 


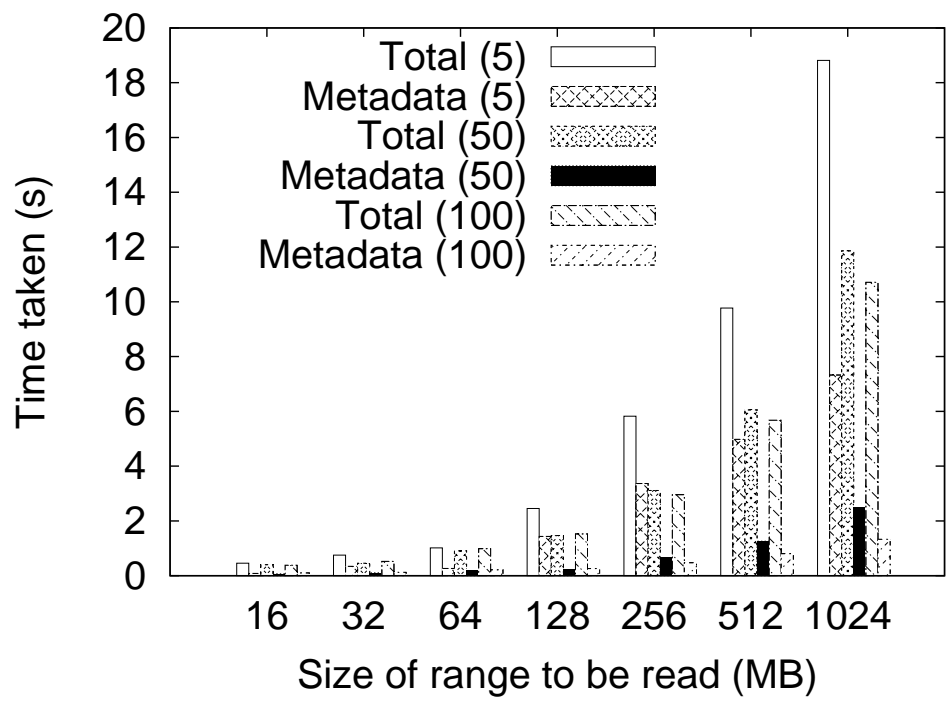

(a) Cost of read accesses when using 5, 50, 100 metadata providers

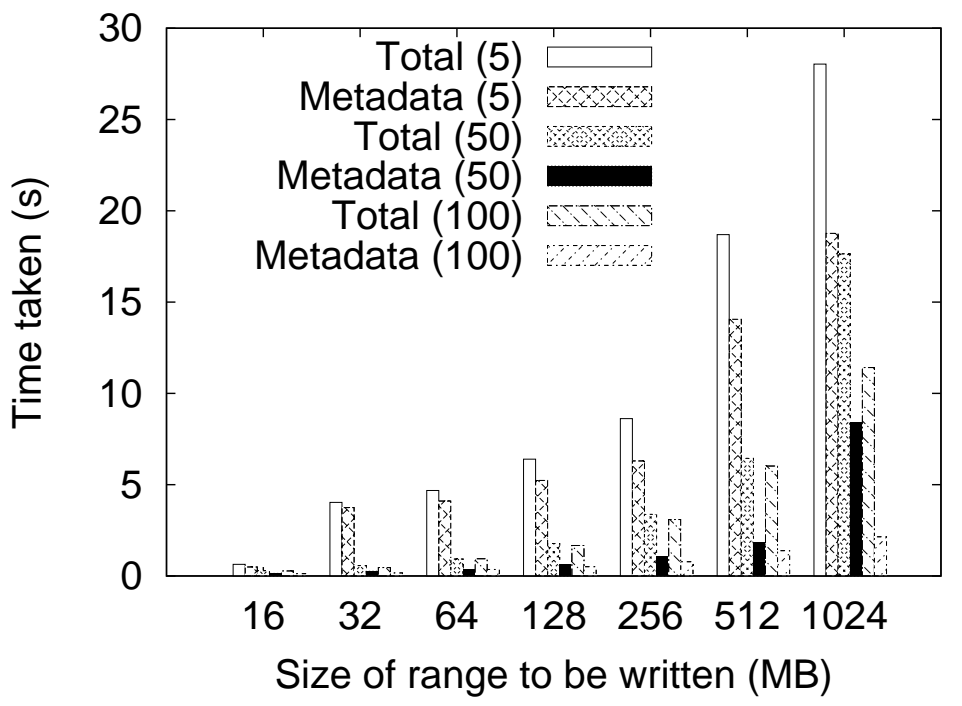

(b) Cost of write accesses when using 5, 50, 100 metadata providers

Fig. 3. Average data access cost for various contiguous segment sizes and a variable number of metadata providers. For each number of metadata providers, we sort out the time needed to manage the metadata. 


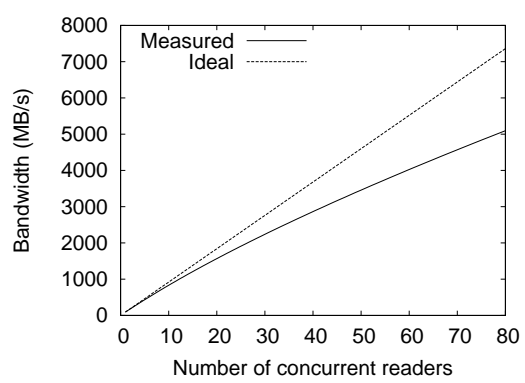

(a) Bandwidth for reads

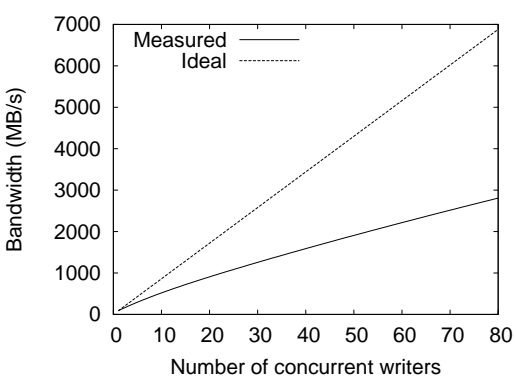

(b) Bandwidth for writes

Fig. 4. Average aggregated bandwidth when varying the number of concurrent clients

the average aggregated bandwidth, both for reads and writes, and compare it to the ideal aggregation of bandwidth obtained from a single reader/writer.

As it can be observed, the fine-grained dispersion of data and metadata allows for high bandwidth under heavy concurrency, especially for reads (Figure 4(a)). Writes suffer from a slight performance penalty because of metadata synchronization (Section 2.4). Contacting different providers and different metadata providers concurrently enables a high degree of load balancing among the network nodes. As such, it makes up for the metadata overhead observed in the first series of experiments.

\section{Conclusion}

We have addressed the problem of efficiently storing massive data of the order of terabytes in a grid distributed environment. Our contribution consists in proposing a data-sharing service allowing to efficiently allocate, access and modify such massive blocks of data in a distributed, multi-user environment. Efficient fine-grain access to arbitrarily small parts of the data is provided thanks to distributed, RAM-based storage of data fragments, while leveraging a DHT-based, natively parallel metadata management scheme. Preliminary experiments performed with our prototype using the Grid'5000 testbed show that our approach scales well, both in terms of storage providers and in terms of concurrency degree.

Our prototype is however a work in progress and definitely demands further refinement. Fault tolerance, which becomes critical in grid environments, is only partially addressed. We currently leverage some fault-tolerance mechanisms provided by the DHT on which we rely for the implementation of some of the entities of our architecture, the metadata providers and the provider manager. This enhances the availability of metadata thanks to the underlying replication used by the DHT. However, the versioning manager, though not under heavy 
load, is still a single point of failure in this preliminary scheme. Besides, data is not replicated: for each page, a single copy is kept on a single provider. In order to improve fault-tolerance, replication-based mechanisms could be envisioned in both cases. To this purpose, we intend to explore the possibility to use selforganizing groups to represent these entities, built on fault-tolerant distributed algorithms for atomic multicast, as in [21].

While targeting database, data-mining and multimedia applications, we have not experimented, yet, with a standard implementation that could use our service. We are considering interfacing our service with the PostgreSQL DBMS, in order to provide an efficient support for snapshot isolation.

\section{References}

1. Douglas, K., Douglas, S.: PostgreSQL. New Riders Publishing, Thousand Oaks, CA, USA (2003)

2. Thomasian, A.: Concurrency control: methods, performance, and analysis. ACM Computing Survey 30(1) (1998) 70-119

3. Nicola, M., Jarke, M.: Performance modeling of distributed and replicated databases. IEEE Trans. on Knowl. and Data Eng. 12(4) (2000) 645-672

4. Jin, R., Yang, G.: Shared memory parallelization of data mining algorithms: Techniques, programming interface, and performance. IEEE Trans. on Knowl. and Data Eng. 17(1) (2005) 71-89

5. Casey, M.A., Kurth, F.: Large data methods for multimedia. In: Proc. 15th Intl. Conf. on Multimedia (Multimedia '07), New York, NY, USA, ACM (2007) 6-7

6. Allcock, B., Bester, J., Bresnahan, J., Chervenak, A.L., Foster, I., Kesselman, C., Meder, S., Nefedova, V., Quesnel, D., Tuecke, S.: Data management and transfer in high-performance computational grid environments. Parallel Comput. 28(5) (2002) 749-771

7. Kunszt, P.Z., Laure, E., Stockinger, H., Stockinger, K.: File-based replica management. Future Generation Computing Systems 21(1) (2005) 115-123

8. Lightweight data replicator. Available at http://www.lsc-group.phys.uwm.edu/ LDR/

9. Chirp protocol specification. Available at http://www.cs.wisc.edu/condor/ chirp/

10. Bassi, A., Beck, M., Fagg, G., Moore, T., Plank, J.S., Swany, M., Wolski, R.: The Internet Backplane Protocol: A study in resource sharing. In: Proc. 2nd IEEE/ACM Intl. Symp. on Cluster Computing and the Grid (CCGRID '02), Washington, DC, USA, IEEE Computer Society (2002) 194

11. Bent, J., Venkataramani, V., LeRoy, N., Roy, A., Stanley, J., Arpaci-Dusseau, A., Arpaci-Dusseau, R., Livny, M.: Flexibility, manageability, and performance in a grid storage appliance. In: Proc. 11th IEEE Symposium on High Performance Distributed Computing (HPDC 11). (2002)

12. Tatebe, O., Morita, Y., Matsuoka, S., Soda, N., Sekiguchi, S.: Grid datafarm architecture for petascale data intensive computing. In: Proc. 2nd IEEE/ACM Intl. Symp. on Cluster Computing and the Grid (Cluster 2002), Washington DC, USA, IEEE Computer Society (2002) 102

13. Honeyman, P., Adamson, W.A., McKee, S.: GridNFS: global storage for global collaborations. In: Proc. IEEE Intl. Symp. Global Data Interoperability - Challenges and Technologies, Sardinia, Italy, IEEE Computer Society (June 2005) 111-115 
14. White, B.S., Walker, M., Humphrey, M., Grimshaw, A.S.: LegionFS: a secure and scalable file system supporting cross-domain high-performance applications. In: Proc. 2001 ACM/IEEE Conf. on Supercomputing (SC '01), New York, NY, USA, ACM Press (2001) 59-59

15. Antoniu, G., Bertier, M., Caron, E., Desprez, F., Boug, L., Jan, M., Monnet, S., Sens, P.: GDS: An Architecture Proposal for a grid Data-Sharing Service. CoreGRID series. In: Future Generation Grids. Springer (2006) 133-152

16. Antoniu, G., Boug, L., Jan, M.: JuxMem: An adaptive supportive platform for data sharing on the grid. Scalable Computing: Practice and Experience 6(3) (November 2005) 45-55

17. Pakin, S., Johnson, G.: Performance analysis of a user-level memory server. In: IEEE Ann; Intl. Conf. on Cluster Computing (Cluster 2007). (September 2007)

18. Merkle, R.C.: A digital signature based on a conventional encryption function. In: Conf. on the Theory and Applications of Cryptographic Techniques on Advances in Cryptology (CRYPTO '87), London, UK, Springer (1988) 369-378

19. Bolze, R., Cappello, F., Caron, E., Daydé, M., Desprez, F., Jeannot, E., Jégou, Y., Lanteri, S., Leduc, J., Melab, N., Mornet, G., Namyst, R., Primet, P., Quetier, B., Richard, O., Talbi, E.G., Touche, I.: Grid'5000: A large scale and highly reconfigurable experimental grid testbed. Int. J. High Perform. Comput. Appl. 20(4) (2006) 481-494

20. Rhea, S., Godfrey, B., Karp, B., Kubiatowicz, J., Ratnasamy, S., Shenker, S., Stoica, I., Yu, H.: OpenDHT: a public DHT service and its uses. In: Proc. 2005 Conf. Applications, Technologies, Architectures, and Protocols for Computer Communications (SIGCOMM '05), New York, NY, USA, ACM (2005) 73-84

21. Antoniu, G., Deverge, J.F., Monnet, S.: How to bring together fault tolerance and data consistency to enable grid data sharing. Concurrency and Computation: Practice and Experience 18(13) (November 2006) 1705-1723 